Nervenarzt 2021 · 92:107-114

https://doi.org/10.1007/s00115-020-01057-x

Angenommen: 15. Dezember 2020

Online publiziert: 22. Januar 2021

(c) Springer Medizin Verlag GmbH, ein Teil von Springer Nature 2021

Endovaskuläre Verfahren haben inzwischen einen festen Platz bei der Behandlung von Hirngefäßerkrankungen, z. B. die Thrombektomie beim Schlaganfall. Neurointerventionelle Materialien, wie Katheter und Stents, und deren Handhabung sind einer permanenten Weiterentwicklung unterworfen, was dauerhaftes Lernen erforderlich macht. Insbesondere bei Notfalleingriffen und Reisebeschränkungen können externe Experten hierzu nicht direkt eingebunden werden. Als Lösung können moderne Streamingtechnologien in entsprechende Ausbildungskonzepte integriert werden, um die Patientensicherheit zu erhöhen.

\section{Neuroendovaskuläre Interventionen im klinischen Alltag}

Neuroendovaskuläre Verfahren spielen eine wichtige Rolle in der Diagnostik und Therapie der meisten Gefäßerkrankungen des Gehirns. Komplexe Gefäßerkrankungen wie Aneurysmen werden mittlerweile häufiger endovaskulär als mit offenen chirurgischen Verfahren therapiert und das mit hohen Sicherheitsund Wirksamkeitsraten [1]. Die Ausweitung endovaskulärer Techniken auf die Behandlung des ischämischen Schlaganfalls hat einer großen Zahl von Patienten weltweit einen bedeutenden Nutzen gebracht. Die endovaskuläre Thrombektomie hat sich dadurch in den letzten Jahren erfolgreich als Goldstandard in der Behandlung von Verschlüssen großer

M. Bechstein · E. Goebell · J. Fiehler

Klinik für Diagnostische und Interventionelle Neuroradiologie, Universitätsklinik Hamburg-Eppendorf, Hamburg, Deutschland

\title{
Remote-Proctoring bei neuroradiologischen Interventionen
}

Hirnarterien der vorderen Zirkulation durchgesetzt [2-4].

Die Indikationen für neuroendovaskuläre Techniken werden möglicherweise zukünftig weitere Krankheitsbilder umfassen. So wird derzeit die Wirksamkeit der Behandlung des chronischen subduralen Hämatoms (cSDH) mittels Embolisation der meningealen Arterien [5-7] und die Behandlung symptomatischer nichtstenotischer Plaques der A. carotis interna $[8,9]$ untersucht.

\section{》) Die beschränkte Zahl der Interventionalisten ist ein Engpass in der Weiterbildung}

Die gestiegene Nachfrage nach endovaskulären Eingriffen bedeutet für Krankenhäuser und akademische Zentren eine infrastrukturelle Herausforderung. So müssen Krankenhäuser, die eine 24stündige Schlaganfallbehandlung anbieten wollen, jederzeit ein Team an endovaskulär ausgebildeten Neuroradiologen und medizinisch-technischen Assistenten vorhalten. Die Zahl der Interventionalisten und die damit verbundenen Möglichkeiten der Ausbildung weiterer Interventionalisten sind jedoch beschränkt, was insbesondere bei Betrachtung im europäischen Maßstab den am häufigsten genannten Engpass bei der Weiterentwicklung der Schlaganfallversorgung darstellt [10].

\section{Aktuelle Strategien zur Deckung des Bedarfes an neuroendovaskulären Interventionen}

Patienten mit intrakraniellen Aneurysmen und arteriovenösen Malformationen sollten primär in neurovaskulären Zentren behandelt und verlaufskontrolliert werden. Die Anwendung endovaskulärer Verfahren bei diesen Indikationen führt $\mathrm{zu}$ einem gestiegenen Bedarf an methodenspezifischer Weiterbildung der vor Ort endovaskulär tätigen Neuroradiologen, etwa in der Benutzung flussumleitender Stents („flow diverter“) oder flussunterbrechender Implantate („flow disruptor"). Diese Techniken ermöglichen inzwischen auch die Behandlung vieler atypisch geformter Aneurysmen. Die betroffenen Patienten können aktuell weitgehend durch die bereits vorhandenen Neuroradiologen behandelt werden.

Anders ist die Situation bei Patienten mit ischämischem Schlaganfall aufgrund eines großen Gefäßverschlusses, die in 13.000 Fällen im Jahr in Deutschland mittels endovaskulärer Thrombektomie (EVT) behandelt werden [11]. Mittlerweile existieren in vielen Regionen Organisationsstrukturen, die die schnellstmögliche Vermittlung betroffener $\mathrm{Pa}$ tienten $\mathrm{zu}$ einem entsprechend qualifizierten neuroradiologischen Team ermöglichen. Weit verbreitet ist das „Dripand-ship“-Konzept: Der Patient wird unter laufender intravenöser tPA(„tissue plasminogen activator")-Therapie in ein neurovaskuläres Zentrum transportiert. Die EVT kann theoretisch auch vor Ort in regionalen Krankenhäusern angebo- 
ten werden. Ein alternatives Konzept ist daher die Aussendung eines Neuroradiologen in ein peripheres Krankenhaus („drip and drive“), um den Patienten vor Ort in der Angiographieeinheit der örtlichen Radiologie zu versorgen [12-14]. Während diese Konzepte vor allem für Metropolregionen geeignet sind, gestaltet sich die endovaskuläre Versorgung von Patienten in ländlicheren Regionen mit langen Transportzeiten weiterhin schwierig. Eine paneuropäische Studie hat entsprechend große geografische Ungleichheiten in der akuten Schlaganfallbehandlung aufgezeigt [10]: Die jährliche Zahl der Thrombektomien schwankte zwischen 0 und etwa 56/1000 ischämischen Schlaganfallpatienten im Jahr 2016.

\section{Neuroendovaskuläre Aus- bildung in der Neuroradiologie}

Die praktische Ausbildung eines Neuroradiologen im vollen Spektrum aller Eingriffe umfasst üblicherweise mehrere Jahre, aber auch allein die thrombektomiespezifische Weiterbildung von Neuroradiologen ist inklusive des Erlernens eines Komplikationsmanagements ein zeitaufwendiger Prozess von 6 bis 12 Monaten [15]. In dieser Zeit sollte ein Neuroradiologe in einem Krankenhaus mit hoher Falldichte zunächst von einem Experten direkt angeleitet und im Verlauf mit zunehmenden Freiheiten supervidiert werden. Dies ist meist regulärer Bestandteil des Weiterbildungskurrikulums in akademischen Zentren. Kleinere Krankenhäuser mit geringem Personalbestand können dagegen ihre Neuroradiologen nur eingeschränkt für eine erforderliche externe Hospitation freistellen. Kann anderweitig kein thrombektomiefähiger Neuroradiologe rekrutiert werden, muss sich das Krankenhaus aus der Akutversorgung von Schlaganfallpatienten abmelden.

Aber auch nach formalem Abschluss der endovaskulären Ausbildung müssen Techniken weiter gelernt und gelehrt werden, nicht zuletzt, weil sich die in den Eingriffen verwendeten Materialien permanent weiterentwickeln. Neue Materialien erfordern oft eine andere Handhabung, die entsprechend einge- übt werden muss. Dies wird zumeist an „Hands-on“-Kursen mit Flussmodellen und Simulatoren absolviert [16].Erfolgen danach die ersten elektiven klinischen Einsätze des neuen Produkts sind neben den technischen Produktspezialisten oftmals zusätzlich externe erfahrene ärztliche Anwender (Proctoren) zugegen, die den lokalen Kollegen mit ihrem spezifischen Wissen unterstützend zur Seite stehen. Ein solches Proctoring ist aber bei akuten Eingriffen (z. B. beim Schlaganfall) organisatorisch nicht darstellbar. Zudem kam es in den letzten Monaten durch COVID-19 (,coronavirus disease 2019“) zu einer massiven Einschränkung der Reisetätigkeit, was ein Proctoring auch bei elektiven Fällen praktisch unmöglich machte.

Eine mögliche Lösung für beide Szenarien ist ein Proctoring unter Verwendung eines externen Kamerasystems (Telementoring/Teleproctoring/ Remote-Proctoring; $[17,18]$ ). Der permanente Zugang zu solchen Möglichkeiten wird inzwischen in globalen Umfragen und Leitlinien ausdrücklich gefordert $[19,20]$.

\section{Telementoring (Remote- Proctoring) bei chirurgischen und interventionellen Eingriffen}

Das Konzept des Telementorings wird in der Chirurgie bereits seit mehr als 20 Jahren eingesetzt, wobei sich insbesondere endoskopische Verfahren hierzu anbieten [21]. Aber auch mikroneurochirurgische Operationen [22] und endovaskuläre Eingriffe $[23,24]$ wurden bereits mit dieser Technologie begleitet.

In einer Vielzahl von Studien wurden verschiedene methodische Ansätze zum Telementoring untersucht. Die Ergebnisse einer aktuellen Metaanalyse legen nahe, dass das Telementoring ein ähnliches Sicherheits- und Wirksamkeitsprofil wie die Unterstützung durch einen vor Ort anwesenden Mentor aufweist [21]. In insgesamt zwölf dieser Studien wurde das Telementoring direkt mit dem Mentoring vor Ort verglichen. So schlussfolgern die Autoren basierend auf den sehr guten Langzeitergebnissen nach Implantation von Aortenstents, dass das Telementoring eine ausgezeichnete Einübung endovaskulärer Fähigkeiten in die Routinepraxis in abgelegenen Gesundheitsversorgungsstandorten ermöglicht [24]. Sieben ( $58 \%$ ) zeigten keinen Unterschied in den Ergebnissen zwischen Telementoring und Vor-Ort-Betreuung. Keine Studie ergab, dass Telementoring zu schlechteren postoperativen Ergebnisse geführt hätte [21].

Eine Anwendung in der interventionellen Neuroradiologie war bis vor Kurzem noch nicht systematisch erfolgt. Eine Bildübertragung aus Angiographieräumen gab es lediglich im Rahmen von Konferenzen mit "live cases" mit aufwendiger Fernsehtechnik. Ein elektronischer Austausch zu akuten Behandlungsfällen fand teilweise mit einfachen kommerziellen Messenger-Diensten statt [25]. Das Interesse an ausgereifteren Methoden zum Remote-Proctoring hat durch COVID19 erheblich zugenommen [19].

\section{Technische Umsetzung für die interventionelle Neuroradiologie}

Die inzwischen allgegenwärtigen $\mathrm{Vi-}$ deostreaminglösungen sind für die nutzerfreundliche Kommunikation vieler Gesprächsteilnehmer optimiert und dafür sehr gut geeignet. Die dadurch vorgegebenen Prioritäten in Entwicklung und Design lassen diese Produkte nicht notwendigerweise für eine Anwendung in der interventionellen Neuroradiologie als geeignet erscheinen, die insbesondere eine Übertragung angiographischer Aufnahmen mit ausreichender Qualität und Stabilität erfordert. Eine entsprechende technische Lösung muss eine hohe Bildqualität, minimale Latenzzeit und eine maximale Zuverlässigkeit garantieren, kann dafür aber mit weniger Gesprächsteilnehmern auskommen. Erreicht wird dies z. B. durch die Verwendung der richtigen Kombination von Streamingtechnologien und eine volle Kontrolle über die Videocodierungsseite, einschließlich der Hardware. Das Vorhandensein geeigneter Sicherheitsmaßnahmen (z.B. die konsequente Verschlüsselung aller Daten) ist eine zentrale Notwendigkeit für einen solchen Einsatz. Auch sind spezifische ergonomische Überlegungen bedeutsam, damit der behandelnde 
Hier steht eine Anzeige.

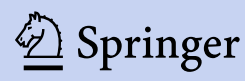


Arzt die volle Kontrolle über eine Streamingsitzung besitzt und sich dennoch voll und ganz auf das jeweilige Verfahren konzentrieren kann.

\section{》) Der Proctor kann sich passwortgeschützt von jedem internetfähigen Computer zuschalten}

Eine solche entsprechende spezifische Onlineplattform ist vor Kurzem entwickelt worden [17] und soll hier kurz exemplarisch beschrieben werden. Das System (Tegus Medical GmbH, Hamburg, Deutschland) besteht aus einer um 360 Grad dreh- und um 180 Grad schwenkbaren „High-Definition“-Netzwerkkamera, die an einem freistehenden, frei im Angiographiesaal beweglichen Stativ montiert ist, und einem mobilen Server, der den Datentransfer und den Onlinezugriff ermöglicht, sowie einer Mikrofon- und Kopfhörereinheit für die Kommunikation (• Abb. 1). Die Bildund Audiodaten werden in Echtzeit verschlüsselt auf die unter strengsten Datenschutzauflagen entwickelte Onlineplattform übertragen. Ein thrombektomieerfahrener Neuroradiologe (Proctor) kann sich passwortgeschützt von jedem internetfähigen Computer einschalten und mittels eines Kopfhörers mit Mikrofon mit dem behandelnden Neuroradiologen kommunizieren, mittels Navigationsschaltflächen die Kamera innerhalb des Angiographiesaales navigieren und auf jeden Punkt im Saal richten und einzoomen, beispielsweise den Angiographiemonitor oder die Hände des behandelnden Arztes. Bei maximalem Zoom ist die Auflösung so hoch, dass die proximalen und distalen Marker sowie die strahlendichten Drähte eines StentRetrievers am Monitor problemlos zu identifizieren sind. Eine zeitliche Verzögerung der Video- oder Audiodaten ist auch bei Verwendung eines gewöhnlichen Breitbandinternetzugangs nur minimal und für den Beobachter kaum wahrnehmbar.

Ein alternatives System zum Telementoring wird von der Firma Olympus (Center Valley, PA, USA) mit dem System

Nervenarzt 2021 · 92:107-114 https://doi.org/10.1007/s00115-020-01057-x

(c) Springer Medizin Verlag GmbH, ein Teil von Springer Nature 2021

\section{Bechstein · E. Goebell · J. Fiehler}

\section{Remote-Proctoring bei neuroradiologischen Interventionen}

\section{Zusammenfassung}

Hintergrund. Endovaskuläre Verfahren haben einen festen Platz bei der Behandlung von Hirngefäßerkrankungen, z. B. die Thrombektomie beim Schlaganfall. Die ständige Weiterentwicklung der hierbei verwendeten Materialien (z. B. Katheter und Stents) fordert von den behandelnden Ärzten ein permanentes Lernen.

Fragestellung. Technische Hilfsmöglichkeiten zur Unterstützung bei neuen neuroendovaskulären Verfahren.

Material und Methode. Integration von Streamingtechnologien in das Ausbildungskonzept von Neuroradiologen.

Ergebnisse. Die Übertragung angiographischer Aufnahmen auf einen entfernten Computerarbeitsplatz in Echtzeit ist mittels spezifischer Streamingtechnologie ortsunabhängig möglich. Hierdurch kann ein neuroendovaskulärer Spezialist geographisch entfernte Interventionalisten bei der Durchführung eines Kathetereingriffes am Gehirn beraten, die Handhabung der verwendeten Materialien überblicken und bei Bedarf anleiten (Remote-Proctoring). Schlussfolgerungen. Insbesondere bei Notfalleingriffen und während Reisebeschränkungen kann durch Zuschaltung eines weiteren neuroendovaskulären Spezialisten per Livestreaming die Patientensicherheit erhöht werden.

\section{Schlüsselwörter}

Thrombektomie · Hirngefäßerkrankung · Telemedizin · Teleproctoring . Telestreamtechnologie

\section{Remote proctoring in neuroradiological interventions}

\section{Abstract}

Background. Endovascular procedures are paramount in the treatment of cerebrovascular diseases, e.g. thrombectomy for stroke. The continuous further development of the devices used for these procedures (e.g. catheters and stents) requires permanent learning by the treating physician.

Objective. Technical support options for new neuroendovascular procedures. Methods. Integration of streaming technologies into the training concept for neuroradiologists.

Results. The transmission of angiographic images to a remote computer workstation in real time is possible independent of location by means of specific streaming technology.
This approach enables a neuroendovascular specialist to advise geographically distant interventionalists when performing catheter interventions of the brain, to oversee the handling of the materials used and to instruct them if necessary (remote proctoring). Conclusion. Especially during emergency interventions and during travel restrictions, patient safety can be increased by connecting to another neuroendovascular specialist via live streaming.

Keywords

Thrombectomy - Cerebrovascular diseases . Telemedicine - Teleproctoring · Telestream technology
MedPresence angeboten. Die Angiographiebilder werden hierbei separat übertragen, wodurch Modifizierungen des Angiographiegerätes erforderlich sind. Andere Anbieter für Telementoringsysteme sind z.B. Avail (Palo Alto, CA, USA) oder Proximie (London, Großbritannien), wobei uns Publikationen im neurointerventionellen Bereich aber nicht bekannt sind. Die frühere Idee, Google Glass (Google, Palo Alto, CA, USA) für Telementoring bei Neuroin- terventionen einzusetzen, scheint sich nicht durchgesetzt zu haben.

\section{Datenschutz und Telementoring}

Die beteiligten Kliniken und technischen Anbieter von Telementoringsystemen unterliegen in Deutschland der europäischen Datenschutzgrundverordnung (DSGVO). So verfügt beispielsweise die Firma Tegus Medical über einen Datenschutzbeauftragten, der die Einhaltung der DSGVO-Standards innerhalb 


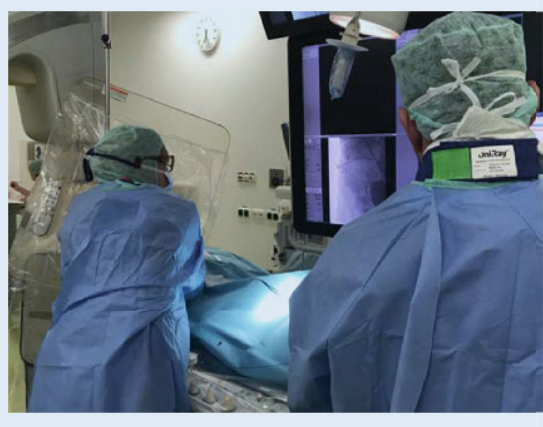

Angiographie Saal (Krankenhaus 1)

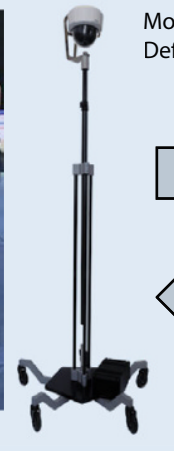

Mobile Einheit mit High

Definition Web-Kamera

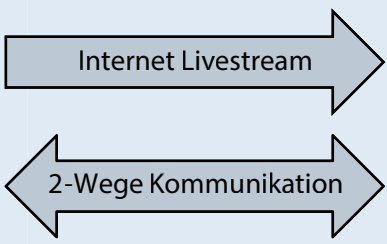

Arbeitsplatz eines spezialisierten endovaskulären Neuroradiologen (Krankenhaus 2, alternativ „home office" oder mobiler Arbeitsplatz mit Laptop)

Abb. 1 ॥ Nutzung der Remote-Streaming-Technologie zur Unterstützung neuroendovaskulärer Eingriffe. Ein neuroendovaskulärer Spezialist überwacht und instruiert beispielsweise eine Schlaganfallthrombektomie, die in einem separaten Krankenhaus durchgeführt wird. Ihm stehen hierbei ein Livestream und Zwei-Wege-Kommunikation zur Verfügung. Der Spezialist kann sich überall befinden, z. B. in einem neurovaskulären Zentrum oder zu Hause mit einem normalen internetfähigen Notebook

des Unternehmens kontinuierlich überwacht und sicherstellt. Mit Vorbereitung der Installation beginnt eine enge $\mathrm{Zu}-$ sammenarbeit mit den IT-Abteilungen und den Datenschutzbeauftragten der Krankenhäuser, um die individuell geforderten Datenschutzinformationen zur Verfügung zu stellen und ggf. technische und organisatorische Anpassungen vorzunehmen. Der behandelnde Arzt entscheidet als einziger uneingeschränkt in jedem Behandlungsfall, wer an der Telementoring/Streamingsitzung teilnimmt und wer eine Zugangsberechtigung zur Ansicht des Eingriffes erhält. Er sollte in einer Nutzen-Risiko-Abwägung wann immer möglich vor dem Streaming das Einverständnis des Patienten einholen. Das Ausblenden des Patientennamens auf dem Angiographiebildschirm sollte grundsätzlich erfolgen.

\section{Experimentelle und praktische Erfahrungen mit dem Remote- Proctoring}

Die technische Durchführbarkeit einer mittels Remote-Proctoring unterstützten Thrombektomie wurde unter Studienbedingungen an einem Angiographiesimulator untersucht [17]. Sechs Radiologen ohne vorherige Thrombektomieerfahrung führten jeweils eine Gesamtzahl von 36 Thrombektomien an einem endovaskulären Simulator der Firma Mentice durch (Mentice VIST G5; Mentice AB, Göteborg, Schweden). Die Szenari- en umfassten verschiedene embolische Großgefäßverschlüsse der vorderen und hinteren Zirkulation. Szenarienabhängig war entweder eine direkte Aspiration des Thrombus, die Platzierung eines StentRetrievers mit Bergung des Thrombus oder ein ergänzendes Stenting erforderlich. Die eingriffsführenden Ärzte wurden hierbei von einem erfahrenen Neuroradiologen angeleitet, der wechselnd nach jedem Szenario entweder direkt mit am Patiententisch stand (konventionelles Proctoring) oder mittels Telestreamtechnologie online von einem anderen Raum zugeschaltet war (Remote-Proctoring). Die primäre Ergebnisvariable war die Zeit bis zur Rekanalisation, gemessen ab dem Zeitpunkt des Einsetzens des ersten Katheters bis zur Wiedereröffnung des verschlossenen Gefäßes. Sekundäre Zielvariablen waren die Zahl gescheiterter Thrombektomien, die Anzahl der für eine erfolgreiche Rekanalisation erforderlichen Versuche, die Handhabungsgenauigkeit des Stent-Retrievers, die Menge des verwendeten Kontrastmittels und die Durchleuchtungszeit. Es wurden keine signifikanten Unterschiede in den Rekanalisationszeiten, der Anzahl gescheiterter Thrombektomien, im Kontrastmittelverbrauch, den Durchleuchtungszeiten oder der Handhabungsgenauigkeit des Stent-Retrievers gemessen. Fragebögen ergaben ebenfalls vergleichbare Ergebnisse für die subjektiv empfundene Sicherheit während des Eingriffes, der Verständlichkeit der Anweisungen und der Zufriedenheit der Behandler mit der manuellen Umsetzung der Anweisungen.

\section{》) Telestreaming ermöglicht ein Proctoring auch bei Notfalleingriffen}

Obwohl diese Studie durch ihre kleine Stichprobengröße und ihren experimentellen Aufbau begrenzt ist, deuten die Ergebnisse klar darauf hin, dass Neuroradiologen bei der Ausführung endovaskulärer Verfahren wie einer notfallmäßigen Thrombektomie von ortsfernen Spezialisten unterstützt werden können.

Erste Erfahrungen aus elektiven neurovaskulären Eingriffen, aber auch bei Notfällen liegen zwischenzeitlich vor [26], bei denen sich zeigte, dass das System sicher einsetzbar ist und die klinischen Erwartungen erfüllt, die an ein Proctoring gestellt werden. Ein weiterer Einsatz bei der Einführung neuer Materialien zur Unterstützung eines weniger erfahrenen Anwenders ist also aussichtsreich. Ob diese Technologie die Behandlung und somit die Prognose von Schlaganfallpatienten in derzeit noch unterversorgten Regionen verbessern kann, bedarf jedoch weiterer umfangreicherer Studien. Eine erste solche Studie wurde gerade von der European Society of Minimally Invasive Neurological Therapy (ESMINT) initiiert. In Kooperation mit drei rumänischen Partnerkrankenhäusern wird die Verwendung des Systems 
Tab. 1 Einsatzmöglichkeiten der Telestreamingtechnologie während neuroendovaskulärer Eingriffe

\begin{tabular}{l} 
Szenario \\
\hline Backup: Komplikationsma- \\
nagement bei der akuten Be- \\
handlung eines Schlaganfalles \\
bei geringerer Erfahrung des \\
lokalen endovaskulären Be- \\
handlungsteams (periphere \\
Krankenhäuser mit geringer \\
"case load“) \\
Erhöhte Patientensicherheit bei \\
der Einführung neuer interven- \\
tioneller Produkte
\end{tabular}

Vordergrunddienst (Facharztniveau) führt endovaskulären Eingriff durch

Komplexer elektiver neuroendovaskulärer Eingriff in einem neuroendovaskulären Zentrum ("Low-incidence-high-risk"-Szenario; z. B. Embolisation einer arteriovenösen Malformation mit mehreren Zuflüssen)

Neuroendovaskuläre Weiterbildung

\section{Potenzielle Vorteile des Tele- Praktische Überlegungen streamings \\ Ein erfahrener Spezialist (z. B. der Lokale Verfügbarkeit eines diensthabende Neuroradiologe Radiologen mit Erfahrung an einem Krankenhaus mit hoher in neuroendovaskulären "case load") kann von ortsfern Eingriffen, lokale Angio- die Thrombektomie in Echtzeit graphieinfrastruktur muss verfolgen und bei Bedarf anleiten vorhanden sein (Remote-Proctoring)}

Erfahrene Anwender können die Einführung ihrer Produkte im klinischen Alltag begleiten (z. B. neuer Katheter)

Hintergrunddienst steht bei Bedarf zur fachlichen Beratung bereit

Weltweite Zuschaltung von Experten zur gemeinschaftlichen Fallbesprechung („expert counsel")

Praktische Demonstration endovaskulärer Fälle in Echtzeit an ein online zugeschaltetes Publikum (E-Fellowship)
Ermöglicht ein Proctoring auch bei Notfalleingriffen

Dienstmodell für einzelne Krankenhäuser oder Krankenhausverbund

Experten müssen vorab informiert werden, Terminabstimmung ggf. auch über Zeitzonen erforderlich

Der Experte (Proctor) befindet sich selbst im Angiographiesaal (,inverse remote proctoring"), Weiterzubildende beobachten den Eingriff via Livestream, kein „Hands-on"-Erlebnis beim Schlaganfall begleitet [27]. Ein weiteres Anwendungsfeld ist die praktische Demonstration endovaskulärer Fälle in Echtzeit an einen online zugeschalteten Trainee (E-Fellowship) als Bestandteil der endovaskulären Ausbildung.

Neben einer ortsunabhängigen Unterstützung der endovaskulären Akutversorgung von Schlaganfallpatienten bestehen noch weitere potenzielle Anwendungen des Livestreaming in der neuroendovaskulären Medizin. Diese sind als Übersicht einschließlich eventueller methodischer Limitationen in • Tab. 1 aufgeführt.

\section{"Neurorobotics" bei neuroradiologischen Interventionen}

Die Einsetzbarkeit der Robotertechnologie wird auch in der endovaskulären Therapie neurovaskulärer Erkrankungen ambitioniert vorangetrieben und kontrovers diskutiert [28, 29]. Unter viel Aufmerksamkeit wurden Anfang des Jahres 2020 drei Studien publiziert, in denen die roboterassistierte enovaskuläre Coil-Embolisation eines intrakraniellen Aneurysmas, diagnostische Angiographien und das Stenting von Karotisstenosen demonstriert wurden [30-32]. Ähnlich dem Da-Vinci-System bei urologischen Operationen führt ein Roboter hierbei mechanisch genau die Aktionen durch, die ein Operateur an einer Bedieneinheit eingibt. Während die Remote-Streaming-Technologie eine räumliche Trennung zwischen dem behandelnden Arzt und einem erfahrenen beratenden Spezialisten erlaubt, kann eine robotergestützte neuroendovaskuläre Behandlung die räumliche Trennung von Patient und Behandler ermöglichen. Dies kann perspektivisch die notfallmäßige Versorgung neurovaskulär erkrankter Patienten weltweit revolutionieren, unabhängig von der lokalen Verfügbarkeit eines neuroendovaskulär erfahrenen Behandlungsteams. Die technischen, finanziellen, infrastrukturellen, datenschutzrechtlichen und nicht zuletzt versicherungsrechtlichen Hürden sind derzeit jedoch so hoch, dass eine Einführung dieser grundsätzlich vielversprechenden Technologie allenfalls langfristig realistisch erscheint. Der bereits jetzt hohe und prognostisch weiter steigende Bedarf an endovaskulär tätigen Neuroradiologen ist ein akutes Problem mit der Notwendigkeit zeitnaher Lösungen.

\section{Fazit für die Praxis}

\section{- Neurointerventionelle Materialien, wie Katheter und Stents, und deren Handhabung werden permanent weiterentwickelt, um die Sicherheit, Wirksamkeit und Handhabbarkeit zu verbessern. Dies erfordert ein kontinuierliches Lernen auf Seiten des Anwenders. \\ - Insbesondere bei Notfalleingriffen und während Reisebeschränkungen können externe Experten (Proctoren) nicht direkt vor Ort zur Unterstützung und Weiterbildung eingebunden werden. \\ - Spezifisch entwickelte Streaming- technologie ermöglicht die digitale Zuschaltung externer Experten während des Eingriffes (Remote- Proctoring) und erhöht so die Patien- tensicherheit.}

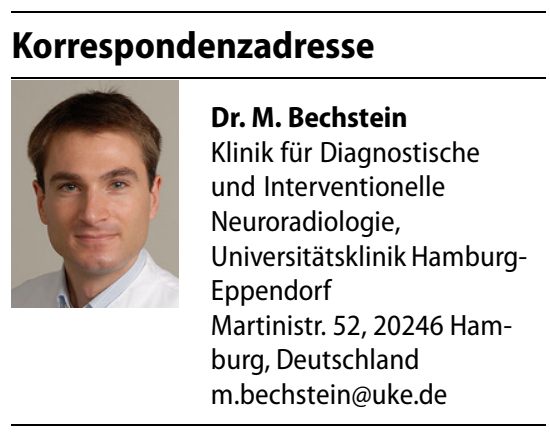

\section{Einhaltung ethischer Richtlinien}

Interessenkonflikt. J. Fiehler hat Forschungsmittel erhalten von: Bundesministerium für Wissenschaft und Bildung (BMBF), Bundesministerium für Wirtschaft und Innovation (BMWi), Deutsche Forschungsgemeinschaft (DFG), Europäische Union (EU), Hamburgische Investitions- und Förderbank (IFB), Medtronic, Microvention, Route92, Stryker. Berater für: Acandis, Bayer, Boehringer Ingelheim, Cereno- 
Hier steht eine Anzeige.

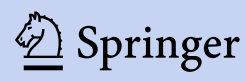


vus, Evasc Neurovascular, MD Clinicals, Medtronic, Microvention, Penumbra, Phenox, Stryker, Transverse Medical. Aktieninhaber: Tegus Medical. M. Bechstein und E. Goebell geben an, dass kein Interessenkonflikt besteht.

Für diesen Beitrag wurden von den Autoren keine Studien an Menschen oder Tieren durchgeführt. Für die aufgeführten Studien gelten die jeweils dort angegebenen ethischen Richtlinien.

\section{Literatur}

1. Riina HA (2019) Neuroendovascular surgery. JNeurosurg 131(6):1690-1701

2. Berkhemer $O A$, Fransen $P S$, Beumer $D$, van den Berg LA, Lingsma HF, Yoo AJ et al (2015) A randomized trial of intraarterial treatment for acute ischemic stroke. N Engl J Med 372(1):11-20

3. Bhogal $P$, Andersson T, Maus V, Mpotsaris A, Yeo $L$ (2018) Mechanical thrombectomy - a brief review of a revolutionary new treatment for thromboembolic stroke. Clin Neuroradiol 28(3):313-326

4. Goyal M, Menon BK, van Zwam WH, Dippel DW, Mitchell PJ, Demchuk AM et al (2016) Endovascula thrombectomy after large-vessel ischaemic stroke: a meta-analysis of individual patient data from five randomised trials. Lancet 387(10029):1723-1731

5. Ban SP, Hwang G, Byoun HS, Kim T, Lee SU, Bang JS et al (2018) Middle meningeal artery embolization for chronic subdural hematoma. Radiology 286(3):992-999

6. Haldrup M, Ketharanathan B, Debrabant B, Schwartz OS, Mikkelsen R, Fugleholm Ket al (2020) Embolization of the middle meningeal artery in patients with chronic subdural hematoma-a systematic review and meta-analysis. Acta Neurochir 162(4):777-784

7. Jumah F, Osama M, Islim Al, Jumah A, Patra DP, Kosty J et al (2020) Efficacy and safety of middle meningeal artery embolization in the management of refractory or chronic subdural hematomas: a systematic review and metaanalysis. Acta Neurochir 162(3):499-507

8. Ospel JM, Singh N, Marko M, Almekhlafi $M$, Dowlatshahi D, Puig J et al (2020) Prevalence of Ipsilateral nonstenotic carotid plaques on computed tomography angiography in embolic stroke of undetermined source. Stroke 51(6):1743-1749

9. Goyal M, Singh N, Marko M, Hill MD, Menon BK, Demchuk A et al (2020) Embolic stroke of undetermined source and symptomatic nonstenotic carotid disease. Stroke 51(4):1321-1325

10. Aguiar de Sousa D, von Martial R, Abilleira S, Gattringer T, Kobayashi A, Gallofre M et al (2019) Access to and delivery of acute ischaemic stroke treatments: a survey of national scientific societies and stroke experts in 44 European countries. Eur Stroke J 4(1):13-28

11. Berlis A, Morhard D, Weber W (2019) On the basis of the deGIR/DGNR register nationwide care for acute Ischemic stroke patients in 2016 and 2017 using mechanical thrombectomy by radiologists and Neuroradiologists. Rofo 191(7):613-617

12. Brekenfeld C, Goebell E, Schmidt H, Henningsen $H_{,}$ Kraemer C, Tebben J et al (2018) "Drip-and-drive": shipping the neurointerventionalist to provide mechanical thrombectomy in primary stroke centers. J Neurointerv Surg 10(10):932-936

13. Seker F, Fiehler J, Mohlenbruch MA, Heimann F, Flottmann F, Ringleb PA et al (2020) Time metrics to endovascularthrombectomy in 3 triage concepts: a prospective, observational study (NEUROSQUAD). Stroke 51(1):335-337

14. Seker F, Mohlenbruch MA, Nagel $S$, Ulfert $C$ Schonenberger S, Pfaff J et al (2018) Clinical results of a new concept of neurothrombectomy coverage at a remote hospital-"drive the doctor". Int J Stroke 13(7):696-699

15. Pierot L, Jayaraman MV, Szikora I, Hirsch JA, Baxter B, Miyachi S et al (2018) Standards of practice in acute ischemic stroke intervention: international recommendations. J Neurointerv Surg 10(11):1121-1126

16. Crossley R, Liebig T, Holtmannspoetter $M$, Lindkvist J, Henn P, Lonn L et al (2019) Validation studies of virtual reality simulation performance metrics for mechanical thrombectomy in ischemic stroke. J Neurointerv Surg 11(8):775-780

17. Bechstein M, Buhk JH, Frölich AM, Broocks $G$, Hanning U, Erler M et al (2019) Training and supervision of thrombectomy by remote live streaming support (RESS) : randomized comparison using simulated stroke interventions. Clin Neuroradiol. https://doi.org/10.1007/s00062-019-00870-5

18. Isaak A, Mallios A, Gürke L, Wolff T (2020) Teleproctoring in vascular surgery to defy COVID19 travel restrictions. Eur J Vasc Endovasc Surg 60(4):623-624

19. Fiehler J, Brouwer P, Diaz C, Hirsch JA, Kulcsar Z, Liebeskind D et al (2020) COVID-19 and neurointerventional service worldwide: a survey of the European Society of Minimally Invasive Neurological Therapy (ESMINT), the Society of Neurolnterventional Surgery (SNIS), the Sociedad Iberolatinoamericana de Neuroradiologia Diagnostica y Terapeutica (SILAN), the Society of Vascular and Interventional Neurology (SVIN), and the World Federation of Interventional and Therapeutic Neuroradiology (WFITN). J Neurointerv Surg 12(8):726-730

20. Khan JM, Khalid N, Shlofmitz E, Forrestal BJ, Yerasi C, Case BC et al (2020) Guidelines for balancing priorities in structural heart disease during the COVID-19 pandemic. Cardiovasc Revasc Med 21(8):1030-1033

21. Erridge $S$, Yeung DKT, Patel HRH, Purkayastha $S$ (2019) Telementoring of surgeons: a systematic review. Surg Innov 26(1):95-111

22. Shenai MB, Tubbs RS, Guthrie BL, Cohen-Gadol AA (2014) Virtual interactive presence for realtime, long-distance surgical collaboration during complex microsurgical procedures. J Neurosurg 121(2):277-284

23. Di Valentino $M$, Alerci $M$, Bogen $M$, Tutta $P$, Sartori F, Marty B et al (2005) Telementoring during endovascular treatment of abdominal aortic aneurysms: a prospective study. J Endovasc Ther 12(2):200-205

24. Porretta AP, Alerci M, Wyttenbach R, Antonucci F, Cattaneo M, Bogen M et al (2017) Long-term outcomes of a telementoring program for distant teaching of endovascular aneurysm repair. JEndovasc Ther 24(6):852-858

25. Calleja-Castillo JM, Gonzalez-Calderon G (2018) Whatsapp in stroke systems: current use and regulatory concerns. Front Neurol 9:388

26. Bechstein M, Elsheikh S, Wodarg F, Taschner CA, Hanning U, Buhk JH et al (2020) Republished: Interhospital teleproctoring of endovascular intracranial aneurysm treatment using a dedicated live-streaming technology: first experiences during the COVID-19 pandemic. J Neurointerv Surg. https://doi.org/10.1136/neurintsurg-2020016722.rep
27. https://www.esmint.eu/training-education/ eastmint/.Zugegriffen: 1.Aug. 2020

28. Albuquerque FC, Hirsch JA, Chen M, Fiorella D (2020) Robotics in neurointervention: the promise and the reality. J Neurointerv Surg 12(4):333-334

29. Goyal M, Sutherland GR, Lama S, Cimflova P, Kashani N, Mayank A et al (2020) Neurointerventional robotics: challenges and opportunities. Clin Neuroradiol 30(2):203-208

30. Mendes Pereira V, Cancelliere NM, Nicholson P, Radovanovic I, Drake KE, Sungur JM et al (2020) Firstin-human, robotic-assisted neuroendovascular intervention. J Neurointerv Surg 12(4):338-340

31. Nogueira RG, Sachdeva R, Al-BayatiAR,Mohammaden MH, Frankel MR, Haussen DC (2020) Robotic assisted carotid artery stenting for the treatment of symptomatic carotid disease: technical feasibility and preliminary results. J Neurointerv Surg 12(4):341-344

32. Sajja KC, Sweid A, AISaieghF, Chalouhi N, Avery MB, Schmidt RF et al (2020) Endovascular robotic: feasibility and proof of principle for diagnostic cerebral angiography and carotid artery stenting. JNeurointerv Surg 12(4):345-349 